\title{
Das Völkerrecht als komplexe Mehr-Ebenen-Struktur ${ }^{1}$
}

Vom Völkerstrafrecht, dem Komplementaritätsprinzip und der Verbindung zum internationalen Menschenrechtsschutz

\section{Kontextualisierung}

\section{Entwicklungsmodelle des Völkerrechts}

Pasquale Stanislao Mancini, Mitbegründer und erster Präsident des Institut de Droit international, hielt im Januar 1851 an der Universität Turin einen seiner berühmtesten Vorträge über die Nationalität als Basis des Völkerrechts: »Della nazionalità come fondamento del diritto delle genti «. ${ }^{2}$ Doch der Nationalstaat europäischer Façon des 19. Jahrhunderts, den Mancini - hier exemplarisch genannt - auch als Persönlichkeit des Risorgimento anstrebte, verwirklichte nicht die Ideale der Aufklärung. Vielmehr kulminierte er im 20. Jahrhundert in den Abgründen zweier Weltkriege. Die klassische Konzeption des Völkerrechts, die den Nationalstaat als vereinzeltes Völkerrechtssubjekt begriff, kam somit den Konflikten der Gegenwart nicht mehr bei.

Zum einen wurde bewusst, dass Frieden nur durch Kooperation erreicht werden konnte. Zum anderen wurde nach 1945 deutlich, dass auch die Kooperation der Staaten allein nicht hinreichen konnte. Denn sie kann auf die bloße Akkumulation staatlicher Interessen hinaus laufen. Der Nationalstaat aber konnte nicht weiter als Selbstzweck begriffen werden, er musste eine dienende Stellung einnehmen. Nicht der Staat, sondern der Bürger muss als Ausgangspunkt des Völkerrechts gesetzt werden. Ihm dient staatliches Handeln. Kommt der Staat dem nicht nach, so muss sein Scheitern durch die internationale Gemeinschaft aufgefangen werden. ${ }^{3}$ Die internationale Gemeinschaft stellt daher mehr als die Akkumulation der Staaten dar, sie besitzt Eigenständigkeit. ${ }^{4}$ Auf diesem Gedanken aufbauend können wir heute eine komplexe Mehr-Ebenen-Struktur ${ }^{5}$ er-

1 Der Aufsatz beruht wesentlich auf Gedanken, die zwischen 2005 und 2007 im Rahmen des gesellschaftswissenschaftlichen Kollegs der Studienstiftung des deutschen Volkes Arbeitsgruppe 1/Völkerrecht (Prof. Dr. Dr. h. c. Christian Tomuschat) unter dem Thema »Schöne neue Welt? Ordnungsmodelle für das 21. Jahrhundert« entstanden. Für Kritik, Anregungen und Diskussionen gilt mein Dank Prof. Dr. Michael Bothe, Prof. Dr. Christoph Möllers, Prof. Dr. Peter Oestmann und Prof. Dr. Dr. h. c. Christian Tomuschat.

2 Vgl. Pasquale Stanislao Mancini, Diritto internazionale, Neapel 1873, S. 5 ff.

3 Vgl. Christian Tomuschat, International Law: Ensuring the Survival of Mankind on the Eve of a New Century, 281 Collected Course of Hague Academy of International Law (1999), S. 237.

4 Vgl. Jan Klabbers, An Introduction to International Institutional Law, Cambridge 2004, S. 39 ff., 335.

$5 \mathrm{Zu}$ einer übergreifenden Darstellung der Herkunft und Verwendung des Begriffes »Ebene « im (völker- und europa-)rechtlichen Kontext vgl. Christoph Möllers, Gewaltengliederung Legitimation und Dogmatik in nationaler und internationaler Rechtsvergleichung, Tübingen 2005, S. 210 ff. Hervorgehoben wird u. a., dass der Ebenenbegriff nicht dazu verleite, innerstaatliche Konzepte (un-)bewusst auf supra- und internationale zu übertragen. 
kennen: Neben den Nationalstaat treten regionale und internationale Organisationen als Ordnungsfaktoren, die nicht den Staat ersetzen, sondern ihn komplementieren. ${ }^{6}$

Daneben sehen wir, wie seit 1945 das Individuum in den Fokus der internationalen Gemeinschaft gerät: ${ }^{7}$ Ihm ist auf universeller Ebene die noch unverbindliche Allgemeine Erklärung der Menschenrechte vom 10. Dezember 1948 gewidmet. Weitere verbindliche Menschenrechtspakte folgten und enthalten teilweise die Möglichkeit der Individualbeschwerde vor quasijustiziellen Ausschüssen, vgl. etwa das Fakultativprotokoll zum Internationalen Pakt über bürgerliche und politische Rechte vom 19. Dezember 1966. Auf regionaler Ebene ist die Durchsetzung der Menschenrechte noch stärker ausgestaltet, gemäß Art. 34 EMRK kann der Einzelne eine Klage gegen einen Vertragsstaat anstrengen.

\section{Bedeutung des Völkerstrafrechts für die Analyse der heutigen internationalen Ord- nung}

Gewiss lassen sich dieser Interpretation der Völkerrechtsentwicklung im 20. Jahrhundert andere, v. a. auf faktische Macht rekurrierende Konzepte entgegen setzen, wie die wieder erstarkende Idee der hegemonialen Ordnung. ${ }^{8}$ Doch erlaubt die Beschäftigung mit dem Völkerstraftrecht gerade einen anderen Rückschluss. Denn hier spiegeln sich eben genannte Aspekte wider: Die von Cassese in ihrer Bezeichnung gedanklich mitangestoßene und im Statut des ICC niedergelegte Idee der Komplementarität des ICC gegenüber nationalen Gerichten, d. h. die Abhängigkeit der Gerichtsbarkeit des ICC davon, dass die nationale Gerichtsbarkeit nicht willens und/oder nicht fähig ist, einzuschreiten, zielt auf eine formelle Mehr-Ebenen-Struktur ${ }^{9}$ ab. Materiell untersagen die Straftatbestände fundamentale Menschenrechtsverletzungen, richten sich dabei jedoch nicht auf den Staat als Handelnden, sondern durchbrechen, jedenfalls auf den ersten Blick, seine Souveränität und greifen das Individuum als Täter heraus. Die Menschenrechte werden daher durch Menschenpflichten gespiegelt. Dies wirft eine weitere Frage auf: Betrachten wir das Völkerrecht als einheitliche Rechtsordnung, dem gemeinsame Prinzipien zugrunde liegen, so können wir die einzelnen völkerrechtlichen Regime nicht als losgelöst voneinander sehen, sondern benötigen eine weiter greifende Konzeptionalisierung. An der Schnittstelle vom Internationalen Menschenrechtsschutz und dem Völkerstrafrecht liegt eine solche Verbindung auf der Hand.

6 Vgl. Klabbers, a. a. O. (Fn. 4), S. 41, 338 ff.

7 Vgl. Louis Henkin, International Law: Politics, Values and Functions, 216 Collected Course of Hague Academy of International Law (1989), S. 208 ff.

8 Vgl. Hermann Mosler, Die Grossmachtstellung im Völkerrecht, Heidelberg 1949, S. 12 ff. und Christian Tomuschat, Multilateralism in the Age of US Hegemony, in: Ronald St. John Macdonald/Douglas M. Johnston (Hrsg.), Towards World Constitutionalism - Issues in the Legal Ordering of the World's Community, Leiden/Boston 2005, S. $31 \mathrm{ff.}$

9 Vgl. Antonio Cassese, Remarks on Scelle's Theory of "Role Splitting" (dédoublement fonctionnel) in International Law, EJIL 1 (1990), S. 210 (210). 


\section{Das Komplementaritätsprinzip als Aspekt der formellen Analyse}

Das in Absatz 10 der Präambel als solches bezeichnete und in Art. 15, 17, 18 und 19 des Römischen Vertrages als Zulässigkeitsvoraussetzung näher ausgeführte Prinzip der Komplementarität lässt sich im historischen Kontext des Völkerstrafrechts abgrenzen. So im Folgenden nach dem Versuch einer begrifflichen Klärung mit früheren Konzepten des Völkerstrafrechts kontrastiert, stellt es den Endpunkt einer Entwicklung dar.

\section{Zum Begriff der Komplementarität}

Komplementarität, wie schon gesagt, bezeichnet die Zuständigkeit des ICC im Falle der Unfähigkeit und/oder Unwilligkeit des Vertragsstaates zur Verfolgung. ${ }^{10}$ Der feine Unterschied zwischen den Begriffen der Subsidiarität und der Komplementarität ist v. a. ein solcher der Zwischentöne: Die Subsidiarität bezieht sich auf eine hierarchisch übergeordnete Ebene, die gegenüber der ihr untergeordneten nachrangig handelt, hinter ihr also grundsätzlich zurücktritt. Der Begriff der Komplementarität hingegen bezieht sich auf ein gleichrangiges Verhältnis im Sinne einer Wechselseitigkeit, in dem sich zwei Ebenen gegenseitig ergänzen und bedingen. ${ }^{11}$ Als solcher wird er auch in der Kommunikationstheorie verwandt. ${ }^{12}$ Die Komplementarität deutet auf eine Zirkularität des Diskurses hin, indem ein Element das andere vervollständigt. Dieses Idealbild allerdings wird durch die Wirklichkeit kontrastiert, in der eine Feindseligkeit nationaler Gerichte gegenüber dem ICC nicht auszuschließen ist. ${ }^{13}$ Interessant ist hierbei gerade der Kniff des Komplementaritätsprinzips: Die staatliche Souveränität ist hier berufen, die Funktionsfähigkeit der internationalen Gemeinschaft zu gewährleisten. Die staatliche Souveränität wird insoweit nicht unterminiert, sondern zweckgebunden erhalten. Solange der souveräne Staat die aufrichtige Strafverfolgung gewährleisten kann, greift die internationale Ebene nicht ein. Erst wenn sie versagt, übernimmt sie seine Zweckbestimmung. Daher finden wir keinen Bruch mit dem klassischen Völkerrecht vor, sondern eine Akzentverschiebung, indem eine weitere Ebene geschaffen,

10 Vgl. Antonio Cassese, International Criminal Law, Oxford 2003, S. 351 ff. und Jann K. Kleffner/Gerben Kor (Hrsg.), Complementary Views on Complementarity - Proceedings of the International Roundtable on the Complementary Nature of the International Criminal Court, Amsterdam 25/26 June 2004, Cambridge 2006.

11 Eine andere Komplementaritätskonzeption findet sich in der Charakterisierung des Alten Reichs als komplementären Reichsstaat. Dabei werden Zuständigkeiten nicht gestuft auf mehrere Ebenen verteilt, sondern von vornherein nur einer Ebene zugestanden. Beide Ebenen zusammen ergeben dann eine einheitliche Staatsgewalt. Vgl. Georg Schmidt, Geschichte des Alten Reichs - Staat und Nation in der Frühen Neuzeit - 1495-1806, München 1999, S. 44. Hier fehlt deutlich das Element der Zirkularität der Kompetenzen. Komplementarität wird daher als Ergänzung in einem einheitlichen Rahmen verstanden und nicht wie hier als wechselseitige Bedingtheit.

12 Vgl. Paul Watzlawick, Menschliche Kommunikation - Formen, Störungen, Paradoxien, 10. Auflage, Bern u. a. 2000, S. 64.

13 Vgl. William A. Schabas, An Introduction to the International Criminal Court, 2. Auflage, Cambridge 2004, S. 85. 
der Staat als solcher aber erhalten wird. Es nimmt daher nicht Wunder, dass sich auch in anderen Mehr-Ebenen-Strukturen ähnliche Prinzipien wiederfinden. ${ }^{14}$ Komplementarität bedeutet in diesem Sinne also vertikale Komplementarität, eben als sich bedingende vertikale Beziehungen.

\section{Zur geschichtlichen Einordnung der Komplementarität}

Das Völkerstrafrecht ist eine Entwicklung des 20. Jahrhunderts. Sein materieller Ansatzpunkt, nämlich die Erfassung von Kernverbrechen, welche die Menschheit als solche treffen, schlägt sich auch in formellen Abgrenzungskriterien insoweit nieder, dass es vom internationalen und transnationalen Strafrecht zu unterscheiden ist. Das internationale Strafrecht, wie es sich in Deutschland etwa in $\$ \S 3 \mathrm{ff}$. StGB findet, stellt nationales Kollisionsrecht dar, das allein einen grenzüberschreitenden Bezug aufweist. Das transnationale Strafrecht ${ }^{15}$ lässt sich am besten anhand der Piraterie erläutern: Hier führte das Faktum der Piraterie auf hoher See, d. h. in hoheitsfreien Gewässern, dazu, dass sich Staaten gezwungenermaßen und aus Eigeninteresse zur Verbrechensbekämpfung zusammentun mussten. ${ }^{16}$ Das Völkerstrafrecht beruht dagegen nicht auf der Zufälligkeit der grenzüberschreitenden oder gemeinsam notwendigen Verbrechensbekämpfung, sondern auf der Einsicht, dass die Nichtbefolgung von Kernnormen die Menschheit universell trifft.

Der Durchsetzbarkeit dieser Kernnormen waren jedoch zunächst Schranken gesetzt.

14 Vgl. Art. 5 EG (Subsidiaritätsprinzip, das sich hier allerdings auf gesetzliche Komeptenzregelungen bezieht und anders ausgestaltet ist) und hierzu Paul Craig/Gráinne de Búrca, EU Law - Text, Cases, and Materials, 3. Auflage, Oxford 2003, S. 134 ff. Zum Vorbild der EG für das zukünftige Völkerrecht vgl. Klabbers, a. a. O. (Fn. 4), S. 14.

Auch innerhalb einer Ebene selbst finden sich solche Anklänge. So verweist die kommunale Selbstverwaltungsgarantie des Art. 28 II GG (die ebenso durch die Landesverfassungen verbürgt wird) auf ein dezentrales Staatsaufbauprinzip, dass den Grundsatz der Subsidiarität zu Gunsten der Gemeinden (als Organe der Exekutive) gegenüber höheren Einheiten festlegt. Vgl. exemplarisch Johannes Dietlein/Martin Burgi/Johannes Hellermann, Öffentliches Recht in Nordrhein-Westfalen, München 2006, S. 79 (mit dem Hinweis, dass Dezentralisierung notwendigerweise Einbußen an Effektivität und Wirtschaftlichkeit mit sich bringe). Auf der nächsthöheren Ebene zwischen den Ländern als Gliedstaaten und dem Bund existiert wiederum legislativ (vgl. Art. 70 I GG) eine rein ergänzende Komplementarität wie sie oben hinsichtlich des Alten Reiches erläutert wurde. Inwieweit jedoch innerhalb einer Ebene Dezentralisierung zur Verselbständigung führen kann, ist umstritten. Trotz der faktischen Existenz föderaler Staaten ist insofern nach wie vor staatstheoretisch bzw. -rechtlich nicht abschließend geklärt, ob innerhalb eines Staatsgebiets weitere Gliedstaaten existieren können oder ob Doppelstaatlichkeit nur qua Völkerrecht möglich ist. Vgl. zu dieser Fragestellung etwa Raymond Carré de Malberg, Contrubition à la théorie générale de l'État, Paris 1920-1922, Neuauflage 1962, S. 68 ff.

15 In einem umfassenderen Sinne erfassen Fischer-Lescano und Teubner das transnationale Strafrecht, vgl. Andreas Fischer-Lescano/Gunther Teubner, Regime-Kollisionen - Zur Fragmentierung des globalen Rechts, Frankfurt a. M. 2006, S. $111 \mathrm{ff}$.

16 Vgl. Antonio Cassese, When May Senior States Officials Be Tried For International Crimes? Some Comments on the Congo v. Belgium Case, EJIL 13 (2002), S. 853 (857 f.). 


\section{a) Durchsetzung kraft Nationalstaat}

Der Versailler Vertrag sah zum einen in Art. 227 vor, Kaiser Wilhelm II. vor einem besonderen Tribunal der Siegermächte zu richten. Die Niederlande kamen jedoch dem Auslieferungsersuchen der Alliierten nicht nach. Zum anderen verpflichtete sich die deutsche Regierung in Art. 228, die Befugnis der Alliierten anzuerkennen, vor ihren Militärtribunalen Kriegsverbrechen zu verfolgen. Auch dies kam nicht zustande. Letztlich wurden vor dem RG zwölf kleinere Fälle verhandelt, von denen sechs mit Freisprüchen endeten. Die nach dem Gerichtsort benannten »Leipziger Prozesse« stellen daher ein Beispiel für gescheiterte Strafverfolgung durch den Nationalstaat dar. ${ }^{17}$

\section{b) Durchsetzung mittels zweier Militärgerichte}

Nach dem 2. Weltkrieg diente die Erfahrung mit den »Leipziger Prozessen« den Alliierten als abschreckendes Beispiel unwirksamer Strafverfolgung. Sie richteten nun in einem ersten Schritt eigene Militärtribunale in Nürnberg und Tokio ein. Das asiatische Tribunal wurde allerdings heftig kritisiert. ${ }^{18}$ Etwas anders - (zur Kritik sogleich) verhielt es sich mit den so genannten »Nürnberger Prozessen«, die zwischen 1945 und 1946 stattfanden. Sie dienten materiellrechtlich als wegweisende Präzedenzfälle des Völkerstrafrechts. Inhaltlich fällt auf, dass der Völkermord als dogmatisch noch nicht herausgearbeitetes Konzept nicht tatbestandlich erfasst wurde, vielmehr wurden Kriegsverbrechen, Verbrechen gegen die Menschheit ${ }^{19}$ und das Verbrechen des Angriffskrieges verfolgt. ${ }^{20}$ Später, nämlich 1948, wurde dann die Völkermordkonvention auch vor dem Hintergrund des Holocaust geschaffen. Der in Deutschland vorwiegend vorgebrachte Kritikpunkt betrifft das Prinzip des nullum crimen et nulla poena sine

17 Vgl. Kreß, Claus, Leipzig - Nürnberg - Rom, FAZ Nr. 229 v. 2. Oktober 2006, S. 10. Wiggenhorn beschreibt die Leipziger Prozesse zum einen als Gegenpol zu den Nürnbergern und zum anderen beide Prozesse als (unbewusste?) Gegenbeispiele zur späteren internationalen Strafverfolgung, insofern als weder die »Verlierer « noch die »Sieger« urteilen dürften. Vgl. Harald Wiggenhorn, Verliererjustiz - Die Leipziger Kriegsverbrecherprozesse nach dem Ersten Weltkrieg, Baden-Baden 2005, insbesondere S. 463 ff. Dass die Leipziger Kriegsverbrecherprozesse scheiterten, liegt aber nicht an ihrer staatlichen, sondern vielmehr nationalstaatlichen Konzeption. In diesem Sinne verweist das Adjektiv »nationalstaatlich « auf Absolutheitsansprüche, d. h. auf eine absolute, losgelöste Macht (vgl. Klaus Schubert/Martina Klein, Das Politiklexikon, 4. Auflage, Bonn 2006, Stichwort »Nationalstaat «). Nur in einem solchen Umfeld kann eine Scheinjustiz überhaupt existieren. Wie unten zu sehen ist, gestattet aber gerade der ICC, die staatliche Ebene miteinzubeziehen, relativiert sie jedoch durch formelle und materielle Missbrauchskontrolle auf überstaatlicher Ebene (diese Gewaltenrelativierung wiederum kann funktional äquivalent zur Gewaltentrennung verstanden werden).

18 Vgl. Cassese, a. a. O. (Fn. 10), S. 332.

19 Arendt stellt treffend heraus, dass die deutsche Übersetzung von »crimes against humanity « nur »Verbrechen gegen die Menschheit« und nicht etwa »Menschlichkeit« lauten kann. Vgl. Hannah Arendt, Eichmann in Jerusalem - A Report on the Banality of Evil, New York u. a. 1994, S. 275.

20 Vgl. Art. II Nr. 1 des Kontrollratsgesetz Nr. 10 (Bestrafung von Personen, die sich Kriegsverbrechen, Verbrechen gegen den Frieden oder gegen die Menschlichkeit [sic] schuldig gemacht haben) vom 20. Dezember 1945. 
lege, d. h. das Verbot retroaktiver Bestrafung. Dieses Argument lässt sich jedoch bei näherer Betrachtung so nicht aufrecht halten: Alle aufgeführten Verbrechen waren inhaltlich schon vorher in völkerrechtlichen Verträgen enthalten oder existieren qua völkerrechtlichem Gewohnheitsrecht. ${ }^{21}$ Die Strafbarkeit vermag sich dann aus daraus abgeleiteten allgemeinen Rechtsgrundsätzen ergeben. Dieser Gedanke schlug sich auch 1950 in Art. 7 II EMRK nieder. ${ }^{22}$

Eigentlich problematisch ist vielmehr die Schaffung eines Militärgerichtes selbst. Auch wenn das Nürnberger Tribunal als solches Akzeptanz gefunden hat, haftet ihm doch bezüglich seiner institutionellen Struktur der Makel der »Siegerjustiz« an. ${ }^{23}$ Es macht sich dabei selbst angreifbar. Dieser Punkt zeigt sich auch bei der näheren Ausgestaltung des zugrunde gelegten Rechts. So wurde es an das common law angelehnt, mit der Folge, dass beispielsweise die Verschwörung zu einem der genannten Verbrechen strafbar war. Der Tatbestand der Verschwörung aber setzt allein eine geistige Handlung voraus ${ }^{24}$ während die kontinentaleuropäische Rechtstradition stets eine körperliche Handlung als Voraussetzung der Strafbarkeit verlangt. Ein wahrhaft internationales Verfahren muss sich aber zugleich mit den verschiedenen Rechtstraditionen auseinandersetzen und auch durch die Rechtsvergleichung ein eigenständiges kreieren. So findet sich im Statut des ICC eine verfahrensrechtliche Verbindung der Grundsätze des civil und des common law wieder. ${ }^{25}$

21 Das Problem der völkerrechtlichen Fragmentierung (vgl. Report of the Study Group of the International Law Commission, finalized by Martti Koskenniemi, Fragmentation of International Law: Difficulties Arising from the Diversification and Expansion of International Law, Helsinki 2007) könnte dabei eine dogmatische Parallele in den Gedanken der historischen Rechtsschule finden, die sich mit der eher staatlichen Rechtsfragmentierung des 19. Jahrhunderts beschäftigte. So ließe sich an dieser Stelle Gewohnheitsrecht auch mit Windscheid als Rechtsarbeit der Jahrhunderte begreifen, die dann eventuell in einem wie im anderen Fall als Rechtserkenntnisquelle zur dogmatischen Durchdringung genutzt werden kann und damit als Ansatzpunkt der Defragmentierung dient. Vgl. Bernhard Windscheid, Die geschichtliche Schule der Rechtswissenschaft, in Paul Oertmann (Hrsg.), Bernhard Windscheid - Gesammelte Reden und Abhandlungen, Leipzig 1904, S. 66 (74 ff.). In eine ähnliche Richtung zielt Lukas Gschwend, Juristische Zeitgeschichte als völkerstrafrechtliche Hilfswissenschaft, Jahrbuch der Juristischen Zeitgeschichte Band 6 (2004/2005), S. 16 (30).

22 Dagegen spielt überpositiv, d. h. auf argumentativ anderem Wege, die Idee der »Radbruchschen Formel « eine Rolle: Radbruch verneint, kurz zusammengefasst, den Rechtscharakter einer scheinbaren Norm im Falle eines krassesten Verstoßes gegen die Gerechtigkeit. Vgl. Gustav Radbruch, Gesetzliches Unrecht und übergesetzliches Recht, SJZ (1946), S. 105 (107). Dabei ist jedoch zu bedenken, dass grundsätzlich das positive Recht die Rechtssicherheit bietet, die den Primat des Rechts vor dem der Politik, d. h. auch vor Willkürherrschaft schützt. Die zu schnelle Annahme von Gerechtigkeitsverstößen kann daher einen der etwaigen Intention widersprechenden Effekt auslösen.

23 Vgl. die abwägende Betrachtung Casseses, a. a. O. (Fn. 10), S. 332 f.

24 Conspiracy ist daher ein so genanntes inchoate crime, vgl. auch Elizabeth A. Martin (Hrsg.): A Dictionary of Law, 5. Auflage, Oxford 2002, S. 107, 245.

25 Dabei sieht Cassese, a. a. O. (Fn. 10), S. 384 ff., das adversatorische Modell lediglich um inquisitorische Züge ergänzt, während Schabas auf das Bonmot eines agreement on common principles and civil behaviour verweist und das Prozessrecht des ICC als hybride Verbindung beider Modelle darstellt, vgl. Schabas, a. a. O. (Fn. 13), S. $117 \mathrm{ff}$. 


\section{c) Durchsetzung mittels der UN}

Die Möglichkeit, internationale Durchsetzbarkeitsmechanismen des Völkerstrafrechts $\mathrm{zu}$ entwickeln und es damit nicht allein materiell auszugestalten, eröffnete sich erst nach dem Kalten Krieg. Anlass war das Versagen der internationalen Gemeinschaft hinsichtlich der Konflikte des ehemaligen Jugoslawien und des Völkermords in Ruanda. Hier dienten die Errichtung des Jugoslawien- und Ruandatribunals (ICTY und ICTR) als Post-Konflikt-Maßnahmen im Sinne des Art. 39 der UN-Charta.

Dabei wurde ein weiter Friedensbegriff zugrunde gelegt, indem Frieden nicht nur negativ als Abwesenheit von Gewalt begriffen wurde, sondern auch positive Aspekte einer funktionierenden Zivilgesellschaft mit umfassen sollte. ${ }^{26}$ Die Stabilisierungsfunktion strafrechtlicher Verfolgung ist dabei nur ein Aspekt. Der geschichtlichen Aufarbeitung und der Fokussierung der Opfer dienen häufig daneben Wahrheits- und Versöhnungskommissionen, die eine, wenn auch rechtliche Bewertungen einschlieBende, stärkere soziale und politische Befriedungsfunktion besitzen. ${ }^{27}$ Hier eröffnet sich eine weitere Strukturdimension der internationalen Ordnung. Sie lässt partizipative Elemente ${ }^{28}$ durch die Beteiligung verschiedener Bevölkerungsgruppen erkennen und weist gleichzeitig auf die Bedeutung privater Akteure hin. Dies verdeutlicht, dass sich Aspekte der völkerrechtlichen Ordnung (so beispielsweise die Einsetzung einer solchen Kommission durch die UN) mit denen der internationalen Beziehungen sowie auch soziologischen und psychologischen Aspekten verbinden. ${ }^{29}$ Auch wenn die

26 Die Resolution 688 vom 5. April 1991 erwähnt dies explizit: »[The Security Council] 1. Condemns the repression of the Iraqi civilian population in many parts of Iraq, including most recently in Kurdish-populated areas, the consequences of which threaten international peace and security in the region«. Allgemein zum Friedensbegriff vgl. Alfred Verdross/Bruno Simma, Universelles Völkerrecht - Theorie und Praxis, 3. Auflage, Berlin 1984, S. 144. Verdross und Simma verweisen darauf, dass, insbesondere bezüglich massiver Menschenrechtsverletzungen, bereits der UN-Charta nicht nur ein negatives Friedenskonzept zugrunde liegt. Parallel zur rechtlichen Analyse, die eher klar abgegrenzte Bereiche als Grundlagen der Friedensstabilisierung herausgreift, beschäftigt sich auch die Politikwissenschaft mit dem Friedensbegriff. Dabei versteht insbesondere Galtung unter dem Konzept des positiven Friedens ein noch weitergehenderes, indem er strukturelle Gewalt und Fragen sozialer Gerechtigkeit mit in sein Blickfeld nimmt. Vgl. Johan Galtung: Gewalt, Frieden und Friedensforschung, in: Dieter Senghaas (Hrsg.), Kritische Friedensforschung. 2. Auflage, Frankfurt a. M. 1971, S. 55-104.

27 Vgl. Cassese, a. a. O. (Fn. 10), S. 9 ff. und Gernd Hankel, Über die Gataca-Justiz in Ruanda, in: Deutsches Institut für Menschenrechte (Hrsg.), Jahrbuch Menschenrechte 2004, Frankfurt a. M. 2003, S. 176 ff.

28 Partizipation kann auch als ein Strang demokratischer Legitimation verstanden werden. Vgl. allgemein Philippe Mastronardi, Allgemeines Staatsrechts als Lehre vom guten und gerechten Staat, Bern 2007, insbesondere S. 270 ff. Zum völkerrechtlichen Kontext vgl. Rosalyn Higgins, Problems and process - international law and how we use it!, Oxford u. a. 2004, S. 111 ff. Für Higgins beinhaltet dabei das interne Selbstbestimmungsrecht politische und öknomische Eigendetermination, d. h. auch partizipative Elemente.

29 Interessant ist hierbei der Gedanke, dass eine interdisziplinäre Kompetenztrennung nicht möglich sei, vgl. Gschwend, a. a. O. (Fn. 21), S. 28. Das erscheint insofern richtig, als eine interdisziplinäre Hierarchie im Sinne von Vertikalität hier nicht möglich ist. Denkbar ist aber eine interdisziplinäre Komplementarität horizontaler Natur, bedingt durch die Überschneidungen der Fachrichtungen. 
rechtliche Struktur als einzige Verbindlichkeit zu erzeugen vermag, ist sie doch auch im Wechselspiel zu anderen Strukturen zu sehen. Dies zeigt sich besonders deutlich am Beispiel der Wahrheits- und Versöhnungskommissionen. So können sie beispielsweise eine kollektive Dimension aufgreifen, während der Vorzug der strafrechtlichen Verfolgung zunächst gerade in der Individualisierung und der Bezugnahme auf einen Täter und bestimmte Vorfälle liegt. Zugleich erlaubt der individuelle Konflikt natürlich Rückschlüsse auf den kollektiven und vice vers $a{ }^{30}$ Daraus kann gefolgert werden, dass sich die strafrechtliche Verfolgung und die Einsetzung einer Wahrheits- und Versöhnungskommission grundsätzlich bedingen und ergänzen sollten.

Beiden Tribunalen wurde jedoch eine Vorrangstellung vor der nationalen Strafverfolgung eingeräumt, ${ }^{31}$ und insofern die nationale Souveränität vollkommen durchbrochen und nicht ergänzt. Hiervon grenzt sich in der Weiterentwicklung das ICC ab. Es besitzt einen weiteren Vorteil in Bezug auf seine Legitimation: Es ist umstritten, ob der Sicherheitsrat mit der Etablierung des ICTY und ICTR ultra vires gehandelt hat. ${ }^{32}$ Ursprünglich als ausführendes Gremium gedacht, erscheint es zweifelhaft, ob die Einrichtung eines Gerichts nicht unter einem »Gesetzesvorbehalt« stand. Dabei ist allerdings zu beachten, dass wie dargestellt die Struktur der internationalen Gemeinschaft nicht der des Staats entspricht, sie also keine weitere Ebene von Staatsgewalt, sondern eine Hoheitsgewalt eigener Art formt. Prägend für nicht nationale Organisationen ist daher auch nicht das Gewaltentrennungssprinzip im klassischen Montesquieuschen Sinne, ${ }^{33}$ vielmehr lassen sich eindeutige Organzuordnungen zu einer Gewalt gar nicht vornehmen. Internationale Organisationen lassen zwischen ihren Organen vielmehr meist Spannungen und Machtverteilungen zwischen dem Einfluss der Mitgliedstaaten und der Eigenständigkeit der Organisation und damit ein System der checks and balances erkennen. ${ }^{34}$ Wenig überraschend sprach sich auch das ICTY im Fall Tadic für die Legitimität seines Bestehens aus. ${ }^{35}$ Der ICC dagegen beruht auf völkerrechtlichem Vertrag und wird daher von diesem Problemkomplex gar nicht betroffen. Seine ver-

30 Vgl. Martti Koskenniemi, Between impunity and show trials, Max Planck UNYB 6 (2002), S. 1 (17 f.).

31 Vgl. Art. 9 ICTY-Statut und Art. 8 ICTR-Statut.

32 Vgl. Knut Ipsen, Völkerrecht, 5. Auflage, München 2004, S. 672.

33 Vgl. Charles de Secondat Bn de la Brède et de Montesquieu, De l'Esprit des lois, 2 Bände, Neuauflage Paris 1995.

34 Diese Machtverhältnis stellt ein internes Kontrollverhältnis dar, das funktional äquivalent der Gewaltentrennung entspricht. Kontrollmechanismen finden sich, dezentralisiert, bereits im klassischen Völkerrecht durch die vertragliche Konzeption, die dem gegenseitigen Interessenausgleich dient. Im modernen Völkerrecht spielt daneben extern die Vertikalität der Mehr-Ebenen-Struktur eine Rolle: Die übergeordnete Eben fungiert dabei auch als Kontrolleinheit. Das damit verknüpfte Folgeproblem der Justiziabilität von Maßnahmen dieser Kontrollentität wird noch weiter unten aufgegriffen. Einen anderen Analyseansatz wählt Möllers: Er abstrahiert zwar die Konzepte der Gewaltentrennung, gliedert aber auch die Betrachtung der überstaatlichen Ebene anhand der drei klassischen Grundfunktionen von Herrschaftsgewalt, d. h. der legislativen, exekutiven und judikativen. Vgl. Möllers, a. a. O. (Fn. 5), S. 253 ff.

35 Vgl. Prosecutor v. Tadic, Appeals Chamber, ICTY, v. 2. Oktober 1995 (http://www.un.org/ icty/tadic/appeal/decision-e/51002.htm, zuletzt aufgerufen am 10. September 2008). 
tragliche Errichtung birgt zugleich den Nachteil, dass echte Universalität erst durch den Konsens aller Staaten erreicht werden kann. Gemildert wird das allerdings durch die Möglichkeit der Einleitung eines Verfahrens kraft einer Verweisung des Sicherheitsrates gemäß Art. 13 lit. b des ICC-Statuts. Die Tatsache, dass die Situation in Darfur dementsprechend (Sicherheitsratsresolution 1593 vom 31. März 2005) verwiesen wurde, nimmt den Angriffen der USA auf den ICC insoweit ihre Schärfe, als sie ihre kritische Haltung zwar weiter aufrechterhalten, aber auch kein destruktives Veto eingelegt haben.

Während der ICC die beiden Tribunale quasi, d. h. nicht als solche, aber als Typus, ablöst und zwar auch insoweit, als seine Jurisdiktion hinsichtlich der Mitgliedstaaten des Römischen Vertrages weder örtlich noch zeitlich begrenzt ist, entstanden mit Unterstützung der UN weitere partiell völkerstrafrechtliche Gerichte. Damit sind die Sondertribunale, wie sie u. a. in Sierra Leone, Kambodscha und Osttimor bestehen, gemeint. Diese Tribunale sind nationale Gerichte, die in einen internationalen Rahmen eingebettet sind, indem sie durch die UN gestützt und gefördert werden. Sie stellen daher ein Bindeglied zwischen den verschiedenen Ordnungen der aufgezeigten MehrEbenen-Struktur dar. Sie verklammern die Ansprüche der Universalität mit denen der Nationalität, so dass ein Dreiklang aus nationaler, hybrider und internationaler Strafverfolgung entsteht.

\section{Das Individuum im Völkerstrafrecht als Aspekt der materiellen Analyse}

\section{Handlungssubjekte}

Das Völkerstrafrecht entkleidet den Staat. Die banale Feststellung, dass der Staat als abstrakte und letztlich gedankliche Entität als solcher nicht handeln kann, sondern zum Handeln eines Organs bedarf, wird im Völkerstrafrecht von der anderen Seite aus gedacht: Die Welt ist konfrontiert mit menschlichem und nicht mit staatlichem Handeln; menschliches Handeln kann als solches für den Staat gedacht werden. Das Zurückziehen hinter den Schild staatlichen Handelns ist daher bei den betroffenen Straftaten nicht möglich. Das Römische Statut erklärt deshalb in seinem Art. 27 die Irrelevanz der Bekleidung eines öffentlichen Amtes. Dieser Grundkonflikt taucht im Völkerrecht an ähnlicher Stelle, wie die Fälle Pinochet ${ }^{36}$ und Congo v. Belgium ${ }^{37}$ zei-

36 Vgl. R v. Bow Street Metropolitan Stipendiary Magistrate, ex parte Pinochet Ugarte, 3 W.L.R. 1456 (H.L. 1998), aufgehoben durch R v. Bow Street Metropolitan Stipendiary Magistrate, ex parte Pinochet Ugarte (No.2), 2 W.L.R. 272 (H.L. 1999), R v. Bow Street Metropolitan Stipendiary Magistrate, ex parte Pinochet Ugarte, 2 W.L.R. 827 (H.L. 1999). (Zugänglich über http://www.publications.parliament.uk/pa/ld/ldjudgmt.htm - zuletzt aufgerufen am 10. September 2008.) Siehe auch Michael Byers, The Law and Politics of the Pinochet Case, Duke Journal of Comparative and International Law 10 (2000), S. 415 ff.

37 Vgl. Case concerning the arrest warrant of 11 April 2000 (Democratic Republic of the Congo v. Belgium), IGH, v. 14. Februar 2002 (http://www.icj-cij.org/docket/index. $\mathrm{php}$ ? $1=3 \& \mathrm{p} 2=3 \& \mathrm{k}=36 \&$ case $=121 \&$ code $=$ cobe $\& \mathrm{p} 3=4-$ zuletzt aufgerufen am 10. September 2008). 
gen, immer wieder auf. Letztlich wird die staatliche Souveränität, im klassischen Sinne verstanden, bedroht. Sie wird aber nicht aufgehoben, sondern umgewandelt. Der Staat wird auf seine Zwecksetzung reduziert, er ist kein Mehr, kein Größeres als ein organisationseinheitliches Mittel zum Zweck. Er bildet die Organisationsstruktur, die es einem Verbund von Menschen erlaubt, eine Ordnung zu schaffen, die ihnen allen dient. Die fundamentalen Anforderungen an diese Ordnung stellt das Völkerrecht auf. ${ }^{38}$ Sollte diese Struktur nun versagen, so trifft das nicht allein die Struktur als solche, sondern auch die dahinter stehenden, agierenden und versagenden Menschen. Schafft die interne Ordnung also Möglichkeiten, Völkermord, Verbrechen gegen die Menschheit oder Kriegsverbrechen zu begehen und erfüllt damit die an sie gestellten Ansprüche nicht, so kann der Einzelne als Handelnder zur Rechenschaft gezogen werden.

Sehr deutlich wird das bei der Problematik um den Straftatbestand der Aggression. Dieser ist zwar in Art. 5 I lit. d des Römischen Vertrages genannt, harrt aber als Warteschleifentatbestand einer weiteren Festsetzung gemäß Art. 5 II i. V. m. 121, 123 desselben Vertrages. Die politische Brisanz des Aggressionstatbestandes liegt zum einen darin begründet, dass sie die schärfste Verurteilung eines Staates über Art. 39 UNCharta beinhaltet und definitorische Unschärfen besitzt. Das zeigt sich u. a. daran, dass der Sicherheitsrat bisher noch nie die Feststellung einer Angriffshandlung getroffen hat, ${ }^{39}$ sondern stets die milderen, aber gleich wirksamen des Friedensbruches und der -bedrohung i. S. d. Art. 39 UN-Charta. Zum anderen aber würde der individualisierte Vorwurf der Aggression im Völkerstrafrecht die klassische Souveränität offensichtlich unterminieren. Denn in einem klassischen Konflikt zwischen zwei Staaten würde nun auch das handelnde Individuum in das Blickfeld rücken. Vor dem Hintergrund moderner Konflikte und Kriegsführung durch Private drängt sich diese Individualisierung aber unter Umständen geradezu auf.

\section{Völkerrechtliche Anforderungen}

Welches sind nun die völkerrechtlichen Anforderungen, auf die das Völkerstrafrecht im Falle der Nichterfüllung reagiert? Das ICC-Statut gibt mit den aufgeführten Straftatbeständen exemplarisch Antwort. Art. 5 I des Statuts listet die Straftaten auf, die in Art. 6 bis 8 weiter ausgeführt werden. Gemäß Art. 9 wurden zudem die so genannten Verbrechenselemente als Auslegungshilfe geschaffen. Art. 5 I lit. a i. V. m. Art. 6 des Statuts stellt den Völkermord unter Strafe und rekurriert dabei wörtlich auf die bereits erwähnte Völkermordkonvention. Art. 5 I lit. b i. V. m. Art. 7 betrifft Ver-

38 Vgl. Louis Henkin, a. a. O. (Fn. 7).

39 Der Sicherheitsrat hat sich der Feststellung einer Angriffshandlung insbesondere auch während der Koreakrise (vgl. Resolution 82 vom 25. Juni 1950) und dem irakischen Überfall auf Kuwait 1990 (vgl. Resolution 660 vom 2. August 1990) enthalten und diese vielmehr als Friedensbrüche erfasst. Da dies dieselben Rechtsfolgen auslöst, sah er sich auch nicht zu der heikleren, aber eventuell nahe liegenderen Bezeichnung gezwungen. 
brechen gegen die Menschheit ${ }^{40}$ und nimmt daher weitestgehend auf den internationalen Menschenrechtsschutz Bezug. Art. 5 lit. c i. V. m. Art. 8 verbietet Kriegsverbrechen und nimmt dabei detailliert Normen des humanitären Völkerrechts auf. Hierbei ist interessant, dass Art. 8, wenn auch in geringerem Maße, neben dem internationalen auch den internen Konflikt erfasst. Alle drei Verbrechen setzen daher kein staatenübergreifendes Tatelement voraus. Vielmehr besitzen sie ein Schwellenelement, d. h. sie verlangen ein Verbrechen von Gewicht und beziehen sich somit nur auf die schwerwiegendsten Verbrechen. Die im ICC-Statut benötigten Schwellenelemente sind dabei folgende: Das Verbrechen des Genozids setzt eine besondere Vorsatzform, einen dolus specialis voraus. ${ }^{41}$ Damit ist grundsätzlich gemeint, dass, während der objektive Tatbestand zwar bestimmte Handlungen voraussetzt, aber nicht fordert, dass die betroffene Gruppe ganz oder teilweise zerstört wurde, der Täter subjektiv eben genau diese Absicht hegt. Das objektive Defizit wird daher mit einem subjektiven Überschuss verknüpft. Die Verbrechen gegen die Menschheit beziehen sich nicht allein auf bestimmte Handlungen, sondern verlangen, dass diese wiederum im Rahmen eines ausgedehnten oder systematischen Angriffs auf die Zivilbevölkerung und in Kenntnis dieses Angriffs begangen werden. Kriegsverbrechen werden vom ICC insbesondere dann verfolgt, wenn sie Teil eines Planes oder einer Politik oder als Teil der Begehung solcher Verbrechen in großem Umfang verübt werden.

Insofern finden sie ihre völkerrechtliche Quelle nicht nur in Vertragswerken, sondern auch im zwingenden Völkergewohnheitsrecht. Sie enthalten somit unumstrittene materielle Kernverbote. Dies verdeutlicht den Wandel des Völkerrechts: Der Staat wird nicht mehr formell als Hülse begriffen, dessen Interna von seiner Souveränität gedeckt sind und der daher auch nur im Falle der Beeinträchtigung eines anderen Staates und damit dessen innerer Struktur zur Verantwortung gezogen werden kann. Vielmehr wird der Staat materiell begriffen und damit werden an seine interne Struktur Anforderungen gestellt sowie auf die hinter dem Staat stehenden Menschen zurückgegriffen, wie oben gezeigt wurde. Insofern trifft ein innerhalb des Staates begangenes Verbrechen auch die internationale Gemeinschaft. ${ }^{42}$

40 Dabei ist aus völkerrechtlicher Perspektive gemäß Art. 128 des ICC-Statuts i. V. m. Art. 33 I des Wiener Übereinkommens über das Recht der Verträge Deutsch keine authentische Sprache. Es fragt sich daher, wie sich die Begriffe der exemplarisch ausgewählten authentischen Sprachen crimes against humanity, crimes contre l'humanité und crímenes de lesa humanidad in die deutsche Sprache übersetzen lassen. Dabei macht Arendt deutlich, dass die gewöhnliche deutsche Übersetzung mit »Verbrechen gegen die Menschlichkeit « untauglich ist: »I have mentioned before the Nuremberg Charter's definition of 'crimes against humanity' as 'inhuman acts' which were translated into German as Verbrechen gegen die Menschlichkeit - as though the Nazis had simply been lacking in human kindness, certainly the understatement of the century.« Vgl. Arendt, a. a. O. (Fn. 19). Zuvor stellt Arendt, auch in Auseinandersetzung mit Jaspers, dar, inwiefern es vielmehr um »crimes against humanity « im Sinne von »crimes against the human status/mankind « gehe. Vgl. Arendt, a. a. O. (Fn. 19), S. 268 f. Dementsprechend ist die deutsche Übersetzung mit »Verbrechen gegen die Menschheit « wohl treffender.

41 Vgl. Schabas, a. a. O. (Fn. 13), S. 38.

42 Vgl. wiederum Louis Henkin, a. a. O. (Fn. 7). 


\section{Menschenrechte und -pflichten}

Für die weitere Analyse herauszugreifen ist insbesondere das Verhältnis von internationalem Menschenrechtsschutz und Völkerstrafrecht. Dabei ist die Bezeichnung Menschenpflicht im Gegensatz zum Menschenrecht zumindest missverständlich bzw. zweideutig.

\section{a) Selbständige Menschenpflichten}

Zum einen können Menschenpflichten selbständig konstruiert werden, indem sie neben den Menschenrechten eine einheitliche Kategorie mit anderem Gehalt bilden. Sie sind insofern Pflichten des Einzelnen gegenüber der Gesellschaft, d. h. binden das Individuum an Kollektivität und nicht etwa Schranken wie sie der Konzeption der Grundrechte des GG zugrunde liegen. ${ }^{43}$ Die afrikanische Banjul - Charta der Menschenrechte und Rechte der Völker statuiert ebensolche Pflichten, indem die materiellen Normen des Teil 1 in Rechte und Pflichten gegliedert werden. Diese Pflichten bestehen gegenüber der Familie, der Gesellschaft und sogar dem Staat sowie weiteren Einheiten und werden als solche v. a. in Art. 27-29 näher ausgeführt. ${ }^{44}$ Sie sind allerdings nicht durchsetzbar. Indem hier Pflichten des Einzelnen gegenüber einem kollektiven Element bestimmt werden, ist es wenig erstaunlich, dass dieser Ansatz noch einen Schritt weiter gedacht wird. So nimmt die afrikanische Menschenrechtscharta in Art. 20-24 auch Menschenrechte der so genannten dritten Dimension auf, d. h. sie formuliert für Völkerschaften kollektive Menschenrechte. ${ }^{45}$ Das wiederum ist anders als die individuelle Pflichtenbindung kritisch zu betrachten: Dogmatisch wird hier nämlich der Staat zugleich als Adressat und als Träger von Menschenrechten konstruiert. Daneben ähneln Menschenrechte dieser Art meist eher politischen Programmsätzen und weisen daher eher Ähnlichkeit zu den konkretisierungsbedürftigen und letztlich zu unbestimmten Staatszielbestimmungen des deutschen GG auf als zu verbindlichen Rechten. ${ }^{46}$

\section{b) Unselbständige Menschenpflichten}

Zum anderen können Menschenpflichten unselbständig auf Menschenrechte verweisen bzw. mit ihnen wechselseitig korrelieren.

So ist zunächst ein Vergleich interessant zwischen Art. 29 der Allgemeinen Erklärung der Menschenrechte, der besagt:

»1. Jeder hat Pflichten gegenüber der Gemeinschaft, in der allein die freie und volle Entfaltung der Persönlichkeit möglich ist. 2. [...]《

und Art. 27 II der Banjul-Charta, der wie folgt lautet:

43 Vgl. Makau Mutua, The Banjul Charter and the African Cultural Fingerprint: An Evaluation of the Language of Duties, Virginia Journal of International Law 35 (1995), S. $344 \mathrm{ff}$.

44 Vgl. U. Oji Umozurike: The African Charter on Human and People's Rights, Den Haag, Boston, London 1997, S. 63 ff.

45 Umozurike spricht anschaulich von »solidarity rights«, a. a. O. (Fn. 44), S. 50 ff.

46 Vgl. Eibe Riedel, Menschenrechte der dritten Dimension, EuGRZ 1989, S. 9 ff. 
»(2) Jedermann übt seine Rechte und Freiheiten unter angemessener Berücksichtigung der Rechte anderer, der kollektiven Sicherheit, der Sittlichkeit und der gemeinsamen Interessen aus. «

Hier wird deutlich, dass die Allgemeine Erklärung der Menschenrechte nur notwendige, sich aus den Menschenrechten selbst ergebende Pflichten fordert. Wenn dem Einzelnen die freie und volle Entfaltung seiner Persönlichkeit möglich sein soll, so muss sie jedem Einzelnen zustehen, woraus sich ergibt, dass die Persönlichkeitsentfaltung des einen ihre Grenze in der des anderen findet und insofern diese Beschränkung auch als Pflicht umformuliert werden kann.

Daneben richtet sich jedes Menschenrecht zumindest ${ }^{47}$ an einen Adressaten, der ebenso spiegelbildlich durch die Menschenrechte verpflichtet wird.

So ist auch die Verwendung des Begriffs Menschenpflicht im Kontext des Völkerstrafrechts zu verstehen. Hier bedeuten die Menschenrechte des Einzelnen zugleich die Pflicht des Staates, ihnen nachzukommen. ${ }^{48}$ Indem aber, wie gezeigt wurde, im Völkerstrafrecht durch die staatliche Souveränität hindurch gegriffen und der handelnde Mensch als Täter erfasst wird, treffen ihn nun eben diese Pflichten.

Als plastisches Beispiel sei die Folter genannt: Folter stellt zum einen ein Verbot des ius cogens dar. Daneben beinhaltet das Folterverbot eine Verpflichtung erga omnes. ${ }^{49}$ Daraus wird deutlich, wie eine fundamentale Verbotsnorm als Anliegen aller Staaten betrachtet wird und damit die Staatengemeinschaft als solche trifft. Zum anderen ist das Folterverbot in zahlreichen universellen als auch regionalen Menschenrechtspakten enthalten ${ }^{50} 1984$ wurde eigens ein Übereinkommen gegen Folter und an-

47 Während in Bezug auf die nationalen Grundrechte des GG stets zwischen zwei Adressaten, nämlich dem Grundrechtsberechtigten und dem -verpflichteten unterschieden wird, vgl. etwa Bodo Pieroth/Bernhard Schlink, Grundrechte - Staatsrecht II, 22. Auflage 2006, S. 28 ff., spielen im Bereich des internationalen Menschenrechtsschutzes die jedenfalls plakativ als solche zu kennzeichnenden Dichotomien zwischen Naturrecht und Positivismus sowie, in diesem Falle etwas im Hintergrund, von Universalismus und Relativismus eine größere Rolle. Werden die Menschenrechte als universell und vor allem als dem Recht vorausgesetzt gesehen, so ist jede menschenrechtliche Normierung lediglich deklaratorisch, vgl. David Sidorsky, Contemporary Reinterpretations of the Concept of Human Rights, in: David Sidorsky (Hrsg.): Essays on Human Rights - Contemporary Issues and Jewish Perspectives, Philadelphia 1979, S. 89. Aus positivistischer Sicht können Menschenrechtsdokumente als konstitutiv verstanden werden (und damit auch als relativistisch, d. h. vom spezifischen Kulturkreis abhängig). Somit existiert danach mit dem Menschenrechtsverpflichteten ein echter zweiter Adressat. Das unterstreicht Kamenka, indem er feststellt: $»$ Rights are claims that have achieved a special kind of endorsement or success $[\ldots] \ll, \mathrm{vgl}$. Eugene Kamenka, Human Rights, People's Rights, in: James Crawford (Hrsg.), The Rights of Peoples, New York u. a. 1988, S. 127. Eine Übersicht gibt auch Antonio Boggiano, Derecho Internacional - Derechos de las Relaciones entre los Ordenamientos Jurídicos y Derechos Humanos, Buenos Aires 2001, S. 79 ff.

48 Vgl. Henry J. Steiner/Philip Alston, International Human Rights in Context - Law, Politics, Morals, 2. Auflage, Oxford 2000, S. 181.

49 Vgl. Barcelona Traction, Light and Power Company Case (Belgium v. Spain), ICJ Reports 1970, S. 3 (32, § 34).

50 Vgl. u. a. Art. 5 der Allgemeinen Erklärung der Menschenrechte, Art. 7 IPBPR, Art. 3 EMRK und Art. 5 II AMRK. 
dere grausame, unmenschliche oder erniedrigende Behandlungen oder Strafen geschlossen. Als Menschenrecht des Einzelnen richtet es sich so gegen den Staat als Verpflichteten. Kommt dieser seiner Verpflichtung durch Tun oder Unterlassen nicht nach und unterlässt er es insbesondere mangels Möglichkeit oder Willigkeit, strafrechtliche Verfolgung aufzunehmen, so schlägt das Verhältnis um. Die Anforderung des Völkerrechts an den versagenden Staat wird nun nicht mehr durch ihn, sondern komplementär durch die internationale Ordnung vollzogen. Ein Mechanismus ist der des Völkerstrafrechts, ein anderer mag in der Rechenschaftsnahme des Staates liegen. So kann beispielsweise Folter ein Verbrechen gegen die Menschheit i. S. d. Art. 7 I lit. f ICC-Statut darstellen. Hier wird nun als Verpflichteter der tatsächlich Handelnde herausgegriffen. Insofern schlägt das Menschenrecht in eine Menschenpflicht des anderen um. In den Fällen, in denen, anders als bei der Folter, nicht schon die amtliche Eigenschaft Definitionsvoraussetzung ist, sondern die Tat auch durch Privatpersonen begangen werden kann, lässt sich die den Täter treffende Menschenpflicht in Bezug setzen zu einem reinen Unterlassen des betroffenen Staates.

\section{Implikationen für die Völkerrechtsordnung durch die Vernetzung materieller Regime - Verhältnis Internationaler Menschenrechtsschutz und Völkerstrafrecht}

\section{Dogmatik des Völkerrechts}

Um ein zukünftiges völkerrechtliches Ordnungsmodell auf der Basis der Analyse bestehender Verhältnisse zu entwerfen, bedarf es - so wird vielfach geschrieben - einer »Konstitutionalisierung « des Völkerrechts. ${ }^{51}$ Diese Konstitutionalisierung darf nicht als staatliche Verfassung verstanden werden, denn, wie bereits gezeigt, entsprechen sich diese beiden Ebenen nicht. Der Begriff der »Konstitutionalisierung « darf v. a. nicht dazu verleiten, völkerrechtliche Fragmentierung als Übel zu begreifen und eine einheitliche Neuordnung als harmonische Lösung zu ersehnen. Der Grund des Übels liegt nicht in den Rechtsquellen, sondern, um mit Savigny zu sprechen, in uns. ${ }^{52}$ Es bedarf daher einer kohärenten Ordnung dogmatischer Natur. Das bedeutet, dass die dog-

51 Vgl. Armin von Bogdandy, Constitutionalism in International Law: Comment on a proposal from Germany, HILJ 47 (2006), S. 223 ff.

52 Vgl. Friedrich Carl von Savigny, Vom Beruf unsrer Zeit für Gesetzgebung und Rechtswissenschaft, Heidelberg 1814 S. 111 und 161 ff. Savigny verlangt drei Voraussetzungen, um das Problem der Rechtsfragmentierung zu behandeln: eine Analyse der Rechtsquellen, einen gut ausgebildeten Juristenstand und Rechtsdurchsetzungsmechanismen. Die Frage der Neustrukturierung durch Kodifikation dringt damit allenfalls als Fomalie in den Hintergrund. Das Problem der Rechtsfragmentierung des 19. Jahrhunderts lässt sich dabei nicht als Blaupause übertragen, enthält aber ein vergleichbares Kernproblem. Rechtsgeschichtliche Forschung ist dabei (als Hilfswissenschaft?) nicht nur inhaltlich gewinnbringend, sondern auf der Metaebene ist eine weitere Vernetzung spannend: Das hier herausgegriffene Beispiel des Völkerstrafrechts lebt in seiner Anwendung von einem Spannungsverhältnis zwischen Jurist und Historiker, vgl. dazu Gschwend, a. a. O. (Fn. 21), insbesondere S. 22 ff. und $33 \mathrm{f}$. 
matische Durchdringung des Rechts viel weiter führt als eine Defragmentierung durch weitere Rechtsquellen.

Worin äußert sich diese? Zum einen besteht nun die staatliche Souveränität in klassischer Selbstherrlichkeit nicht mehr, sondern wurde durch Zweckbindung und - im Falle des Versagens - durch Übernahme der Staatengemeinschaft sowie durch partielle Übertragung gewandelt. Zum anderen treten weitere Völkerrechtssubjekte auf: Internationale Organisationen als teilweise Verkörperung der Staatengemeinschaft sowie Individuen. Daher besitzt das Völkerrecht schon jetzt keine eindimensionale Struktur mehr, es wird nicht mehr allein bestimmt durch die Staaten als Rechtssubjekte und gleichzeitige -objekte. Die dargestellte Mehr-Ebenen-Struktur birgt aber entsprechende Gefahren: Das derzeitige Völkerrecht ist changierend, weil es im Wandel begriffen ist. Eine Erkenntnis scheint genauso möglich wie die Gegenteilige. So mag man im Völkerstrafrecht den Beweis eines funktionierenden Durchsetzungsmechanismus wie - dargestellt - erkennen, der Macht durch Recht bricht. ${ }^{53}$ Dass die Macht dennoch letztlich entscheidend ist, mag der Kritiker durch den Hinweis untermauern, dass sich das System bisher noch nicht erwiesen habe und vor allem im Bereich der Exekution Schwachstellen dadurch aufweise, dass es wiederum auf staatliche Kooperation angewiesen sei. ${ }^{54}$ Dies allerdings lässt außer Betracht, dass oberste Gerichte häufig zur Durchsetzung auf ihre Autorität rekurrieren müssen. So kann das BVerfG im innerstaatlichen Bereich lediglich gemäß $§ 35$ BVerfGG bestimmen, wer seine bindenden Entscheidungen wie vollstrecken soll, es besitzt aber nicht selbst Vollzugsorgane. Auch der EGMR kann seine Entscheidungen auf regionaler Ebene nicht durchsetzen. Die Vertragsparteien verpflichten sich lediglich gemäß Art. 46 EMRK zur Durchführung, die das Ministerkomitee überwacht. Befolgt werden die Urteile dennoch. Daneben haben es auch das ICTY und das ICTR bewältigt, Verfahren durchzuführen.

Das Beispiel zeigt, wie sehr Analysen des derzeitigen Völkerrechts letztlich in ihrer Gewichtung per se als Prognoseentscheidungen ausfallen müssen. Je nachdem, welche Indikatoren zur Untermauerung der eigenen Interpretation herangezogen werden, lassen sich äußerst divergierende Auffassungen vertreten. Daher sind Aussagen über die jetzige Eigenart des Völkerrechts angesichts seiner dynamischen und teilweise unstimmigen Struktur weniger - wenn auch vermeintlich - Aussagen über die Gegenwart als Prognosen dahingehend, welche der Strömungen sich durchsetzen könnte. Um aber das Mehr-Ebenen-System verwirklicht zu sehen, muss es rechtlich kohärent sein.

\section{Gefahren durch Legalitätslücken}

Andernfalls drohen wiederum rechtlich nicht $\mathrm{zu}$ tolerierende Legalitätslücken. Als Beispiel mögen folgende Vorfälle dienen: Durch die Sicherheitsratsresolution 1267 vom 15. Oktober 1999 wurden dem afghanischen Talibanregime finanzielle Sanktionen auferlegt. Dazu wurde ein Sanktionskomitee errichtet, dessen Aufgabe es u. a.

53 Zum Verhältnis von Macht und Recht vgl. Oscar Schachter, International Law in Theory and Practice, London 1991, S. 5 ff.

54 Vgl. bereits Walther Burckhardt, Die Unvollkommenheit des Völkerrechts, Bern 1923, v. a. S. $4 \mathrm{ff}$. 
war, eine Liste von terroristisch vernetzten Personen zu erstellen. Diese trafen u. a. durch die erweiternde Sicherheitsratsresolution 1390 vom 28. Januar 2002 Sanktionen wie das Einfrieren von Kapital und Reiseverbote. Dies wiederum musste im Rahmen der GASP innerhalb der Europäischen Union mittels der Verordnung 881/2002 (später ergänzt um die Verordnung 561/2003) umgesetzt werden. Betroffene Personen klagten nun zunächst vor ihren Heimatgerichten und später im Wege der Nichtigkeitsklage gemäß Art. 230, 225 I EG vor dem Gericht erster Instanz. Dieses sah sich aber nicht fähig, über die Geltung von Sicherheitsratsresolutionen zu entscheiden. Allein das hier nicht als verletzt angesehene ius cogens bilde dafür eine Grenze. ${ }^{55}$ Durch ein nicht vollständiges Mehr-Ebenen-System, in dem auf internationaler Ebene ein verbindlicher Überwachungsmechanismus fehlt, entsteht so eine Legalitätslücke. Die Fälle dieser Art konnten bisher nur über diplomatische Wege im Sanktionskomitee gelöst werden, wobei zurzeit Rechtsmittelverfahren gemäß Art. 225 I EG i. V. m. Art. 56 ff. des Protokolls über die Satzung des Gerichtshofs vor dem EuGH anhängig sind und sich der Generalanwalt bereits in zwei Schlussanträgen im Januar diesen Jahres für die Aufhebung der früheren Urteile, auch unter Berufung auf den gemeinschaftsrechtlichen Grundrechtsschutz, aussprach. ${ }^{56}$

Fraglich bleibt, wie Systemfehler dieser Art rechtlich behoben werden können. Politische Vorstöße de lege ferenda scheinen, wie Reformversuche jüngst zeigten, auf UN-Ebene aussichtslos.

\section{Lösungsansätze}

Einerseits kann hierbei auf schleichende Prozesse der Verrechtlichung hingewiesen werden. In Reaktion auf die Geschehnisse errichtete das, durch die Sicherheitsratsresolution 1267 vom 15. Oktober 1999 eingesetzte, Sanktionskomitee im Jahr 2006 Möglichkeiten zur Überprüfung und zur Schaffung eines transparenteren Verfahrens. Die Sicherheitsratsresolution 1730 vom 19. September 2006 kreierte im UN-Sekretariat eine Stelle, die Beschwerden der Betroffenen annimmt und bearbeitet. Auf diese Weise können politische Prozesse mit der Zeit immer stärker zu Recht gerinnen. ${ }^{57}$ Dogmatisch ähnlich verhält es sich im Bereich des internationalen Menschenrechtsschutzes, wo weichere Mechanismen wie die des Staatenberichtes und der -beschwerden sich zumindest im Bereich der bürgerlichen und politischen Menschenrechte um den härteren Mechanismus der Individualbeschwerde ergänzten. ${ }^{58}$ Selbst die durch

55 Vgl. Rs. T-306/01 - Yusuf und Al Barakaat International Foundation / Rat und Kommission, Rs. T-315/01 - Kadi / Rat und Kommission, Rs. T-253/02 - Ayadi / Rat, zugänglich über http://curia.europa.eu/jurisp/cgi-bin/form.pl?lang=de (zuletzt aufgerufen am 10. September 2008).

56 Vgl. Rs. C-402/05 P - Kadi / Rat und Kommission sowie Rs. C-415/05 P-Al Barakaat International Foundation/Rat und Kommission, zugänglich über http://curia.europa.eu/ jurisp/cgi-bin/form.pl?lang=de (zuletzt aufgerufen am 10. September 2008).

57 Zur Beschreibung des Rechts als geronnene Politik vgl. Dieter Grimm, Recht und Politik, Juristische Schulung (1969), S. 501 ff.

58 S. etwa das Fakultativprotokoll zum IPBPR und das Fakultativprotokoll zum Übereinkommen zur Beseitigung jeder Form von Diskriminierung der Frau. 
den ECOSOC gemäß Art. 68 UN-Charta errichtete politische und nicht quasijustiziell agierende Menschenrechtskommission (jetzt Menschenrechtsrat) besitzt durch ECOSOC-Resolutionen 1235 (XLII vom 6. Juni 1967) und 1503 (XLVIII vom 27. Mai. 1970) zum einen die Möglichkeit, Menschenrechtsverletzungen zu untersuchen sowie auf sie zu reagieren, und konnte zum anderen ein Beschwerdeverfahren hinsichtlich systematischer Menschenrechtsverletzungen einrichten.

Andererseits können sich Auffangmechanismen entwickeln. So machte das Gericht erster Instanz in vergleichbaren Fällen, welche die Umsetzung der Sicherheitsratresolution 1373 vom 28. September 2001 betrafen, Unterschiede: Diese, ähnliche Maßnahmen wie die der bereits genannten Resolutionen anordnende Resolution, lässt anders als die vorherigen die Identifikation der betroffenen Personen und das Verfahren in der Hand der umsetzenden Staaten bzw. hier der supranationalen Organisation. Somit sah sich das Gericht auch hier bereits befähigt, die umsetzende Verordnung 2580/2001 voll zu überprüfen (der dadurch entstehende Konflikt zwischen den Ebenen wurde bereits oben aufgezeigt). ${ }^{59}$

\section{Insbesondere: Vernetzung materieller Regime}

Darüber hinaus vermag rechtliche Vernetzung aus völkerrechtlichen Fragmenten ein einheitliches und kohärentes System zu schaffen. Dazu bedarf es einer Ordnung des Völkerrechts dogmatisch übergreifender Art. Während das allgemeine Völkerrecht weitestgehend so strukturiert ist, stehen die einzelnen völkerrechtlichen Regime teilweise unverbunden nebeneinander. Gerade das Begreifen der völkerrechtlichen Wechselwirkungen ermöglicht es aber, ein einheitliches Ordnungsmodell zu erkennen. Berührungsmomente müssen dazu erkannt, Begriffe einheitlich verwandt werden. Als Beispiel diente hier das Zusammenspiel vom internationalen Menschenrechtsschutz und dem Völkerstrafrecht, indem das Verhältnis von Menschenrecht und -pflicht analysiert wurde. In einem ganz anderen Bereich mag etwa das Verhältnis vom internationalen Flüchtlings- zum Menschenrechtsschutz herausgegriffen werden: Kann etwa die in Art. 1 des Genfer Abkommens über die Rechtsstellung der Flüchtlinge von 1951 zur Definition des Begriffs »Flüchtling« erforderliche Furcht vor Verfolgung in eine Furcht vor einer Menschenrechtsverletzung umformuliert werden $?^{60}$

\section{Ergebnis}

Auf der Basis der komplexen Mehr-Ebenen-Struktur des Völkerrechts wurde das Völkerstrafrecht beispielhaft analysiert. Formell prägt das Prinzip der Komplementarität

59 Vgl. Rs. T-228/02 - Organisation des Modjahedines du peuple d'Iran / Rat, zugänglich über http://curia.europa.eu/jurisp/cgi-bin/form.pl?lang=de (zuletzt aufgerufen am 21. Juli 2008). Vgl. insgesamt zu diesem Komplex Chia Lehnardt, European Court Rules on UN and EU Terrorist Suspect Blacklists, ASIL Insight January 31, 2007 (http://www.asil.org/insights/ 2007/01/insights070131.html, zuletzt aufgerufen am 10. September 2008).

$60 \mathrm{Vgl}$. Leonie Vierck, Relating refugee and human rights (law) politics, Transatalantic Student Forum (http://www.tsf-berlin.de/member\%20publications/Vierck\%20refugee\%20and $\% 20$ human\%20rights\%20politics.pdf, zuletzt aufgerufen am 10. September 2008). 
das ICC-Statut und erläutert eben jene Struktur, indem grundsätzlich der Staat völkerrechtliche Anforderungen zu gewährleisten hat, dabei aber nur zweckdienlich ist, so dass im Falle seines Versagens die internationale Gemeinschaft seine Aufgaben übernimmt. Materiell wurde dabei zugleich die Rolle des Individuums näher betrachtet. Indem der Staat nunmehr zweckdienlich ist, ermöglicht dies zugleich, das menschliche Handeln hinter dem staatlichen zu betrachten und dabei dem Einzelnen so Verantwortlichkeit aufzuerlegen. Darauf aufbauend wurden die Wechselwirkungen exemplarisch zwischen dem internationalen Menschenrechtsschutz und dem Völkerstrafrecht fokussiert. Die Menschenrechte können als eine Anforderung des Völkerrechts an die staatliche Ordnung gesehen werden. Zugleich erfassen sie damit die innerhalb dieser Ordnung agierenden Individuen. Im Falle des staatlichen Versagens ist daher ein direkter Zugriff auf sie möglich. Dadurch rückt das zu schützende Individuum wiederum in den Kern der völkerrechtlichen Aufmerksamkeit. Ihm dienen letztlich sowohl der Staat als auch die internationale Gemeinschaft. Zugleich deutet diese Vernetzung von internationalem Menschenrechtsschutz und Völkerstrafrecht auf einen weiteren Problemkreis hin. Sollte die benannte Mehr-Ebenen-Struktur als solche für das gesamte Völkerrecht als Ordnungsmodell zukunftsweisend sein, so bedarf es jedenfalls einer kohärenten völkerrechtlichen Dogmatik, die nach und nach ihre Regime zu einer Einheit zusammenfassen kann. Dieser Text möchte lediglich methodisch begründen, warum es dieser Analyse gerade auch im Hinblick auf die Mehr-Ebenen-Struktur bedarf, die dann in weiteren Schritten erfolgen sollte. ${ }^{61}$

61 Zu Fragen der Fragmentierung vgl. zum einen den Report Koskenniemis, a. a. O. (Fn. 21) sowie ausführlich die Autoren der so genannten Frankfurter Schule: Andreas Fischer-Lescano/Gunther Teubner, a. a. O. (Fn. 15) und Sonja Buckel/Ralph Christensen/Andreas Fischer-Lescano (Hrsg.), Neue Theorien des Rechts, Stuttgart 2006. 